\title{
Robust control with finite frequency specification for uncertain discrete linear repetitive processes
}

\author{
Wojciech Paszke • Olivier Bachelier
}

Received: 28 July 2012 / Revised: 25 October 2012 / Accepted: 22 November 2012 /

Published online: 14 December 2012

(C) The Author(s) 2012. This article is published with open access at Springerlink.com

\begin{abstract}
This paper is dedicated to the study of robust stability and controller synthesis for discrete linear repetitive processes with polytopic uncertainty. In the robust control domain, conditions based on parameter dependent Lyapunov functions are proposed in order to reduce the conservatism related to uncertainty problems. The solution is a class of Lyapunov functions that depends in a polytopic way on the uncertain parameters and that can be derived from linear matrix inequality conditions. Nevertheless, in many cases in practice, the frequency range of reference signals, noises and disturbances are known beforehand. Therefore, performing controller synthesis in the full frequency range is not practically suited and may introduce conservatism to some extent. Based on generalized Kalman-YakubovichPopov Lemma, a finite frequency controller is derived for uncertain discrete linear repetitive processes which are the most investigated class of $2 \mathrm{D}$ systems. Hence, the designer can specify a frequency range where the prescribed control performance is required, where, for example, this range could be determined by inspection of frequency spectrums of the available signals.
\end{abstract}

Keywords Linear repetitive processes · Parameter-dependent Lyapunov functions · Kalman-Yakubovich-Popov Lemma

This work is partially supported by Ministry of Science and Higher Education in Poland, Grant No. N N514 636540 .

\footnotetext{
W. Paszke $(\bowtie)$

Institute of Control and Computation Engineering, University of Zielona Góra, Ul. Podgórna 50, 65-246 Zielona Góra, Poland

e-mail: w.paszke@issi.uz.zgora.pl

O. Bachelier

University of Poitiers, LIAS-ENSIP, Bâtiment B25, 2 rue Pierre Brousse, B.P. 633,

86022 Poitiers Cedex, France

e-mail: Olivier.Bachelier@univ-poitiers.fr
} 


\section{Introduction}

In the recent years, a considerable amount of attention has been paid to stability and stabilization of linear systems. Numerous results have been presented, many of them being formulated in terms of linear matrix inequalities (LMIs) (see Boyd et al. 1994; El Ghaoui and Niculescu 1999 and the references therein for example). The LMI techniques have become the center of attention of the control community for several reasons. Firstly, the solution to the problem formulated in the LMI fashion can be solved through efficient numerical techniques based on convex optimization (Boyd et al. 1994). Secondly, the basic control problem like stability and stabilization can be relatively easily extended to involve robustness analysis and more control performance specifications using $\mathscr{H}_{2}$ and $\mathscr{H}_{\infty}$ norms. Therefore, in the domain of robust analysis and robust control synthesis for uncertain linear systems, most of the results have an LMI characterization. However, the most direct approach to derive LMI form of the robust control problem is to use a common Lyapunov matrix over the whole uncertainty domain. This obviously can lead to quite conservative results in many practical problems. Hence, to reduce the conservatism inherent to this approach the parameter-dependent Lyapunov functions (PDLFs) have been successfully applied—see (Gahinet et al. 1996; Peaucelle and Arzelier 2001; Ramos and Peres 2001; Trofino 1999) for background in using PDLFs for solving robust control problems.

Unfortunately, the main focus in using PDLFs is on classical, i.e.1D, systems and there is only a little number of results in the area of 2D linear systems (Hmamed et al. 2008) and linear repetitive processes (LRPs) (Cichy et al. 2011; Rogers et al. 2007). The importance of providing those results in the domain of LRPs is motivated by wide range of applications for modeling, analysis and synthesis with the LRPs framework (Azevedo-Perdicoulis and Jank 2012; Rabenstein and Steffen 2012; Wu et al. 2011), especially for long-wall coal cutting, metal rolling (Rogers et al. 2007), interconnected systems (Cichy 2008) and designing of iterative learning control (ILC) schemes (Bristow et al. 2006; Cichy et al. 2011; Rogers et al. 2007). This is mostly why LRPs are the most investigated class of $2 \mathrm{D}$ systems.

According to the importance of LRPs, the paper provides the required results. Particulary, it is shown that robust stability analysis and controller synthesis via PDLFs of discrete LRPs becomes possible through efficient numerical techniques based on LMIs. Also sometimes the frequency ranges of signals are known beforehand so that, for these cases, analysis and controller design in a full frequency range may introduce conservatism to some extent. Therefore, due to those practical requirements, we will develop the approach for direct treatment of multiple frequency domain specifications on the controlled LRP in various frequency ranges. The presented approach is based on applying the generalized Kalman-YakubovichPopov (KYP) lemma that allows us to establish the equivalence between frequency domain inequalities (FDIs) for a transfer-function and an LMI defined in terms of its state space realization (Iwasaki and Hara 2005). Note that there is one result which can be considered as an extension of the generalized KYP to the context of $n \mathrm{D}$ models (Bachelier et al. 2008) but this work is not specifically adapted for LRPs.

In particular, the main contribution of the paper is to provide the LMI characterization for stability along the pass and then extend it to include finite frequency specifications and PDLFs. The key tool is based on applying extended LMI characterizations of considered problems. In particular, we make extensive use of projection lemma (Gahinet and Apkarian 1994) as it is done in recently published papers (Feng et al. 2010; Pipeleers et al. 2009). We can find results for LRPs in Paszke and Bachelier (2009), where the solution is given for quite conservative case only. The motivation behind extended LMI characterization is the possibility to introduce extra matrix variables which allows us to separate the Lyapunov 
matrices from the LRP state-space model matrices. Also, these new matrix variables can decrease the degree of conservatism in some practical cases.

The paper is organized as follows. Section 2 gives the background in discrete LRP stability anaysis and provides LMI conditions for stability along the pass over the finite frequency range via KYP lemma. Section 3 presents the result on controller design for LRPs that is then extended to the case of uncertainty in Sect. 4. Section 5 gives an example to illustrate the effectiveness of the proposed method. Finally, conclusions are given in Sect. 6.

Throughout this paper, the null matrix and the identity matrix with appropriate dimensions are denoted by 0 and $I$, respectively. Moreover, matrix inequalities are considered in sense of Löwner, i.e. the notation $X \succeq Y$ (respectively $X \succ Y$ ) means that the matrix $X-Y$ is positive semi-definite (respectively, positive definite). In large matrix expressions, the symbol $(\star)$ replaces terms that are induced by symmetry. Expression $\operatorname{sym}\{\mathbf{M}\}$ stands for the symmetric matrix $M+M^{T}$ and $\rho(\cdot)$ denotes the spectral radius of its matrix argument. Finally, the superscript $*$ denotes the complex conjugate transpose of a matrix.

Extensive use is made of the following result, known as Elimination Lemma or Projection Lemma the proof of which can be found in Gahinet and Apkarian (1994).

Lemma 1 Given a symmetric matrix $\Gamma \in \mathbb{R}^{m \times m}$ and two matrices $\Lambda$, $\Sigma$ of column dimension $m$, there exists a matrix $W$ such that the following LMI holds

$$
\Gamma+\operatorname{sym}\left\{\Lambda^{T} W \Sigma\right\} \prec 0,
$$

if and only if the following two projection inequalities are satisfied

$$
\Lambda^{\perp^{T}} \Gamma \Lambda^{\perp} \prec 0, \Sigma^{\perp^{T}} \Gamma \Sigma^{\perp} \prec 0 .
$$

In addition, to derive the new set of LMI conditions for analysis and synthesis of LRPs we apply the following result of Iwasaki and Hara (2007).

Lemma 2 For a discrete linear time-invariant system with transfer-function matrix $G(z)$ and frequency response matrix

$$
G\left(e^{j \theta}\right)=C\left(e^{j \theta} I-A\right)^{-1} B+D,
$$

the following inequalities are equivalent:

i) the frequency domain inequality

$$
\left[\begin{array}{c}
G\left(e^{j \theta}\right) \\
I
\end{array}\right]^{*} \Pi\left[\begin{array}{c}
G\left(e^{j \theta}\right) \\
I
\end{array}\right] \prec 0, \quad \forall \theta \in \Theta,
$$

where $\Pi$ is a given real symmetric matrix that describes some properties of $G(z)$, e.g. bounded realness or positive realness, and $\Theta$ denotes the following frequency ranges

\begin{tabular}{|c|c|c|c|}
\hline & low frequency range & middle frequency range & high frequency range \\
\hline$\Theta$ & $|\theta| \leq \theta_{l}$ & $\theta_{1} \leq \theta \leq \theta_{2}$ & $|\theta| \geq \theta_{h}$ \\
\hline
\end{tabular}

ii) the LMI

$$
\left[\begin{array}{cc}
A & B \\
I & 0
\end{array}\right]^{T} \Xi\left[\begin{array}{cc}
A & B \\
I & 0
\end{array}\right]+\left[\begin{array}{cc}
C & D \\
0 & I
\end{array}\right]^{T} \Pi\left[\begin{array}{cc}
C & D \\
0 & I
\end{array}\right] \prec 0
$$


where $Q \succ 0, P$ is a symmetric matrix and the matrix $\Xi$ is partitioned as

$$
\Xi=\left[\begin{array}{l|l}
\Xi_{11} & \Xi_{12} \\
\hline \Xi_{12}^{*} & \Xi_{22}
\end{array}\right]
$$

and specified as follows:

- for the low frequency range

$$
\Xi=\left[\begin{array}{c|c}
\Xi_{11} & \Xi_{12} \\
\hline \Xi_{12}^{*} & \Xi_{22}
\end{array}\right]=\left[\begin{array}{c|c}
-P & Q \\
\hline Q & P-2 \cos \left(\theta_{l}\right) Q
\end{array}\right],
$$

- for the middle frequency range

$$
\Xi=\left[\begin{array}{c|c}
\Xi_{11} & \Xi_{12} \\
\hline \Xi_{12}^{*} & \Xi_{22}
\end{array}\right]=\left[\begin{array}{c|c}
-P & e^{j\left(\theta_{1}+\theta_{2}\right) / 2} Q \\
\hline e^{-j\left(\theta_{1}+\theta_{2}\right) / 2} Q & P-\left(2 \cos \left(\left(\theta_{2}-\theta_{1}\right) / 2\right)\right) Q
\end{array}\right],
$$

- and for the high frequency range

$$
\Xi=\left[\begin{array}{c|c}
\Xi_{11} & \Xi_{12} \\
\hline \Xi_{12}^{*} & \Xi_{22}
\end{array}\right]=\left[\begin{array}{c|c}
-P & -Q \\
\hline-Q & P+2 \cos \left(\theta_{h}\right) Q
\end{array}\right] .
$$

\section{Linear repetitive processes and their stability}

LRPs are one of the most important classes of 2D linear systems of both industrial and algorithmic interest. The essential unique characteristic of such processes is a series of sweeps, termed passes, through a set of dynamics defined over a fixed finite duration known as the pass length and here denoted by $\alpha$. On each pass an output, termed the pass profile, is produced which acts as a forcing function on, and hence contributes to, the next pass profile. This characteristic shows that LRPs have clear 2D system structure and hence it is natural to exploit links between 2D linear systems and LRPs.

Following (Rogers et al. 2007), the state-space model of a discrete LRP has the following form over $0 \leq p \leq \alpha-1, k \geq 0$

$$
\begin{aligned}
x_{k+1}(p+1) & =A x_{k+1}(p)+B_{0} y_{k}(p)+B u_{k+1}(p), \\
y_{k+1}(p) & =C x_{k+1}(p)+D_{0} y_{k}(p)+D u_{k+1}(p),
\end{aligned}
$$

where $\alpha<+\infty$ denotes the pass length, and on pass $k \geq 0 x_{k}(p) \in \mathbb{R}^{n}$ is the state vector, $u_{k} \in \mathbb{R}^{r}$ is the control signal vector and finally $y_{k}(p) \in \mathbb{R}^{m}$ is the pass profile vector (output).

To complete the process description, it is necessary to specify the boundary conditions, that is, the state initial vector on each pass and the initial pass profile. In the forthcoming analysis, no loss of generality arises from assuming that $x_{k+1}(0)=0, k \geq 0$, and $y_{0}(p)=$ $f(p), 0 \leq p \leq \alpha-1$, where the entries in the vector $f(p) \in \mathbb{R}^{m}$ are known functions. For ease of presentation, no further explicit reference is made to the boundary conditions in this paper.

Several sets of necessary and sufficient conditions for stability of discrete LRPs of the form considered here are known (Rogers et al. 2007). From the practical point of view, the most important are those which guarantee asymptotic stability or stability along the pass. Asymptotic stability guarantees a bounded sequence of pass profiles (output signals) for a bounded initial pass profile over the finite and fixed pass length $\alpha$, whereas stability along the pass is stronger since it requires this property uniformly, that is, for all possible values of the pass length and hence, it is not surprising that asymptotic stability is a necessary condition 
for stability along the pass. According to the strong structural links between 2D systems and LRPs, extensively used conditions are those for stability along the pass given in terms of the corresponding $2 \mathrm{D}$ characteristic polynomial defined as

$$
\mathscr{C}\left(z_{1}, z_{2}\right)=\operatorname{det}\left(\left[\begin{array}{cc}
I-z_{1} A & -z_{2} B_{0} \\
-z_{1} C & I-z_{2} D_{0}
\end{array}\right]\right),
$$

where $z_{1}, z_{2} \in \mathbb{C}$ are the reciprocals of $z$-transform variables in the along the pass and passto-pass directions respectively, see (Rogers et al. 2007) again for the details concerning these transform variables and, in particular, how to avoid technicalities associated with the finite pass length, which define a $2 \mathrm{D}$ transfer-function matrix for these processes. Then, it is known that stability along the pass holds if, and only if,

$$
\mathscr{C}\left(z_{1}, z_{2}\right) \neq 0:\left(z_{1}, z_{2}\right) \in \bar{U}^{2},
$$

where $\bar{U}^{2}=\left\{\left(z_{1}, z_{2}\right):\left|z_{1}\right| \geq 1,\left|z_{2}\right| \geq 1\right\}$. The difficulty with this condition lies in verifying them for a given example since it is necessary to work with a polynomial in two indeterminates $z_{1}$ and $z_{2}$. The computational difficulties can be simplified by applying the results of Huang (1972) where it is shown that the stability condition given by the above lemma is equivalent to checking the conditions involving one-variable polynomials only. Then, using the results of Boland and Owens (1980), we can formulate the necessary and sufficient condition for stability along the pass in the following form.

Lemma 3 (Rogers et al. 2007) A discrete LRP of (9) is stable along the pass if, and only if,

i) $\rho\left(D_{0}\right)<1$,

ii) $\rho(A)<1$,

iii) all eigenvalues of $G\left(z_{1}\right)=C\left(z_{1} I-A\right)^{-1} B_{0}+D_{0}$ have modulus strictly less than unity $\forall\left|z_{1}\right|=1$.

In terms of checking the conditions of the above result, the first two pose no problems. However, the third requires eigenvalue computation for all points on the unit circle in the complex plane. One way of avoiding the computational load generated is to use tests that only require computation for a subset of points on the unit circle, termed gridding, but the resulting test is sufficient but not necessary. However, this can be avoided by applying KYP lemma, as it was shown in Paszke et al. (2009), Paszke et al. (2011). In particular, the condition iii) of Lemma 3 can be rewritten in view of KYP lemma because this condition is recognized as bounded realness property of the transfer function $G\left(z_{1}\right)$. Hence, we can obtain the corresponding LMI characterization of stability along the pass.

Lemma 4 (Paszke et al. 2012) A discrete LRP described by (9) is stable along the pass if (if and only if in the SISO case) there exist matrices $R \succ 0, S \succ 0, Q \succ 0$ such that the following LMIs are feasible

$$
\begin{gathered}
D_{0}^{T} R D_{0}-R \prec 0, \\
A^{T} S A-S \prec 0, \\
{\left[\begin{array}{cccc}
-Q & Q A^{T} & Q C^{T} & 0 \\
A Q & -Q & 0 & B_{0} \\
C Q & 0 & -I & D_{0} \\
0 & B_{0}^{T} & D_{0}^{T} & -I
\end{array}\right] \prec 0 .}
\end{gathered}
$$


It should be noted that each LMI in the system (11)-(13) has its own interpretation in view of engineering practice. Namely, the LMI (11) is the asymptotic stability condition. The LMI (12) is the requirement that the first pass profile is uniformly bounded with respect to the pass length. Finally, the LMI (13) means that each frequency component of the initial pass profile is attenuated from pass to pass. Hence, this result is preferred as the base in solving various engineering control problems in contrary to the most popular result in LRPs community, where the following Lyapunov function candidate

$$
V(k, p)=x_{k+1}^{T}(p) P_{1} x_{k+1}(p)+y_{k}^{T}(p) P_{2} y_{k}(p),
$$

is used to formulate the stability along the pass $\left(P_{1} \succ 0\right.$ and $P_{2} \succ 0$ are the matrices to be found). For more details on this technique applied in stability test and controller design for LRPs, see Rogers et al. (2007) and references therein.

Even if the result of Lemma 4 seems to be efficient in checking stability along the pass, in practice, the initial pass profiles have finite frequency spectra rather than in entire frequency domain. Hence the development of stability and stabilization conditions in finite frequency domain is significative for practical applications of LRPs.

The objective is now to develop new stability condition which gives us a possibility to extend it to design procedures with finite frequency range specifications for control of uncertain LRPs.

\subsection{Stability along the pass over the finite frequency range}

In this section we give a very important result which formulates a new condition for stability along the pass of LRPs over the finite frequency range and it will be a basis for the development of our main result. To proceed, it is routine to show that since we choose the matrix $\Pi$ in (3) and (4) as

$$
\Pi=\left[\begin{array}{rr}
I & 0 \\
0 & -I
\end{array}\right],
$$

then $\forall \theta \in \Theta$, i.e. for considered frequency range defined as in Lemma 2, we have

$$
G\left(e^{j \theta}\right)^{*} G\left(e^{j \theta}\right)<I, \quad \forall \theta \in \Theta,
$$

which is just

$$
\bar{\sigma}\left(G\left(e^{j \theta}\right)\right)<1, \quad \forall \theta \in \Theta
$$

where $\bar{\sigma}(\cdot)$ denotes the maximum singular value and $G\left(e^{j \theta}\right)=C\left(e^{j \theta} I-A\right)^{-1} B_{0}+D_{0}$ is a frequency response matrix of (9). According to the known fact that $\bar{\sigma}(\cdot)$ provides the upper bound for the eigenvalues of a matrix, we have

$$
\rho\left(G\left(e^{j \theta}\right)\right) \leq \bar{\sigma}\left(G\left(e^{j \theta}\right)\right)
$$

where equality occurs for SISO systems only since $G\left(e^{j \theta}\right)$ is then a scalar function. This means that in this case only the resulting stability test can be a necessary and sufficient one and otherwise sufficiency is only guaranteed. Based on the above inequality, we can reformulate the condition $\mathrm{ii}$ ) of Lemma 3 over finite frequency ranges to obtain an approach that is more practically relevant and can be applied for our purposes. 
Making use of Lemma 2 for specific frequency range $\Theta$, inequality (3) with (14) is directly converted into (see Iwasaki and Hara 2005 for more details)

$$
\left[\begin{array}{cc}
A & B_{0} \\
I & 0
\end{array}\right]^{T} \Xi\left[\begin{array}{cc}
A & B_{0} \\
I & 0
\end{array}\right]+\left[\begin{array}{cc}
C & D_{0} \\
0 & I
\end{array}\right]^{T} \Pi\left[\begin{array}{cc}
C & D_{0} \\
0 & I
\end{array}\right] \prec 0,
$$

where the matrix $\Xi$ is given by (5) and it is the only matrix which the blocks of depend on chosen frequency range, i.e. low, middle or high frequency range, as it is given by (6), (7) and (8) respectively. Also, Lemma 2 shows that the blocks of $\Xi$ involve terms with $Q \succ 0$ and a symmetric matrix $P$. Hence, the inequality (16) cannot be directly exploited to for control law design because it can involve product terms involving $P$ or $Q$ and the controlled LRP state-space model matrices $A$ and $B_{0}$ for any frequency range. The following result establishes the required transformation.

Theorem 1 A discrete LRP of the form (9) is stable along the pass over a finite frequency range $\theta \in \Theta$ if there exist matrices $S \succ 0, Q \succ 0, P \succ 0$ and $W$ such that the following LMIs are feasible

$$
A^{T} S A-S \prec 0
$$

$$
\left[\begin{array}{cccc}
\Xi_{11} & \Xi_{12}-W & 0 & 0 \\
\Xi_{12}^{*}-W^{T} & \Xi_{22}+A^{T} W+W^{T} A & W^{T} B_{0} & C^{T} \\
0 & B_{0}^{T} W & -I & D_{0}^{T} \\
0 & C & D_{0} & -I
\end{array}\right] \prec 0,
$$

where according to Lemma 2 , the matrices $\Xi_{11}, \Xi_{12}, \Xi_{22}$ form a matrix $\Xi$ of the form (5) and are chosen according to the specific frequency range $\Theta$, i.e. for low, middle or high frequency range, as it is given by (6), (7) and (8) respectively.

Proof To prove the above theorem, it is required to show that if LMIs (17) and (18) are found feasible then LMIs (11)-(13) hold too. First of all, in view of LMI (18), it is straightforward to see that condition (11) is redundant and hence can be removed due to the fact that LMI (18) implies

$$
\left[\begin{array}{rr}
-I & D_{0}^{T} \\
D_{0} & -I
\end{array}\right] \prec 0,
$$

which is immediately equivalent to $D_{0}^{T} D_{0}-I \prec 0$. Obviously, this last inequality guarantees that $\rho\left(D_{0}\right)<1$ and hence it is equivalent to LMI (11). Next, the first LMI in the above theorem are the same as (12) in Lemma 4 and follow immediately from classical Lyapunov stability theory for discrete linear systems. To obtain the inequality (18), i.e. to separate the matrix $\Xi$ from the LRP process model matrices, observe that for the finite frequency range, i.e. when the matrix $\Xi$ has form as in (5), the inequality (16) can be converted into

$$
\left[\begin{array}{ccc}
A^{T} & I & 0 \\
B_{0}^{T} & 0 & I
\end{array}\right]\left[\begin{array}{ccc}
\Xi_{11} & \Xi_{12} & 0 \\
\Xi_{12}^{*} & \Xi_{22}+C^{T} C & C^{T} D_{0} \\
0 & D_{0}^{T} C & D_{0}^{T} D_{0}-I
\end{array}\right]\left[\begin{array}{cc}
A & B_{0} \\
I & 0 \\
0 & I
\end{array}\right] \prec 0,
$$

which is of the form of the first inequality in (2) on introducing the notation

$$
\Lambda^{\perp}=\left[\begin{array}{cc}
A & B_{0} \\
I & 0 \\
0 & I
\end{array}\right], \Gamma=\left[\begin{array}{ccc}
\Xi_{11} & \Xi_{12} & 0 \\
\Xi_{12}^{*} & \Xi_{22}+C^{T} C & C^{T} D_{0} \\
0 & D_{0}^{T} C & D_{0}^{T} D_{0}-I
\end{array}\right] .
$$


Obviously, taking $\Lambda^{\perp}$ as it is defined in (20), we immediately get $\Lambda=\left[\begin{array}{lll}-I & A & B_{0}\end{array}\right]$. Consequently, in order to use the result of Lemma 1 we have to find a matrix $\Sigma^{\perp}$ that satisfies the second inequality of (2). It can be seen that by taking $\Sigma=\left[\begin{array}{lll}0 & I & 0\end{array}\right]$ we get

$$
\Sigma^{\perp}=\left[\begin{array}{ll}
I & 0 \\
0 & 0 \\
0 & I
\end{array}\right]
$$

and the second inequality in (2) becomes

$$
\Sigma^{\perp^{T}} \Gamma \Sigma^{\perp}=\left[\begin{array}{cc}
\Xi_{11} & 0 \\
0 & D_{0}^{T} D_{0}-I
\end{array}\right] \prec 0 .
$$

Indeed, by Lemma 2 we see that for all considered frequency ranges $\Xi_{11}=-P$ and hence it is easy to find a symmetric matrix $P$ that satisfies $-P \prec 0$. Hence, it requires $P \succ 0$. Also, it is obvious that $D_{0}^{T} D_{0}-I \prec 0$ holds since (18) is satisfied. Application of Lemma 1 implies that (19) is feasible if and only if

$$
\left[\begin{array}{ccc}
\Xi_{11} & \Xi_{12} & 0 \\
\Xi_{12}^{*} & \Xi_{22}+C^{T} C & C^{T} D_{0} \\
0 & D_{0}^{T} C & D_{0}^{T} D_{0}-I
\end{array}\right]+\operatorname{sym}\left\{\Lambda^{T} W \Sigma\right\} \prec 0,
$$

holds. Finally, after application of Schur's complement formula, the inequality (21) is transformed into (18) and the proof is completed.

Remark 1 It has to be pointed out that the generalized KYP lemma given in Lemma 2 requires the matrix $P$ to be only symmetric. Hence there is no constraint on the sign definiteness of $P$. This is due to the fact that the generalized KYP lemma does not invoke stability. In other words, we can have a FDI which does not induce stability and therefore $P$ can be non-positive (see Bachelier et al. 2004; Bachelier and Mehdi 2006 for more details on this issue). However, it can be easily shown that the LMI (18) in the above lemma is in fact a constraint on $\mathscr{H}_{\infty}$ norm and hence we have to impose stability because $\mathscr{H}_{\infty}$-norm is defined (and makes sense) for stable transfer functions only. Therefore we need to have $P \succ 0$.

Furthermore, as it was indicated, these conditions become necessary and sufficient for SISO processes (i.e. when $D_{0}$ in (9) is a scalar) and therefore in this case they eliminate the conservatism of already known stability conditions. Also, the relevance of LMI (18) can be illustrated by plotting the eigenvalue loci generated by eigenvalues of $G\left(e^{j \theta}\right)$ per frequency $\theta$ and ensuring that no curve intersects the unit circle.

\subsection{Extended LMI characterization for stability along the pass}

In this section we propose possible extensions to Theorem 1 by applying Lemma 1 . The main aim is to provide an additional degree of freedom by introducing the auxiliary slack variables and by keeping the process matrices independent of the Lyapunov matrix. The impact is mostly on controller synthesis procedures for uncertain processes where PDLFs can be used to reduce conservatism in comparison with the currently used standard LMI based synthesis procedures. Among presented conditions, we choose only one, i.e. the extension 2 , for further developments because it seems to be the best suited for our problem where the common auxiliary matrix variable is used for both considered LMIs. However, we cannot conclude that the LMI extension used in this paper is definitely better than the others for all practical cases, and therefore we introduce some alternative conditions which may actually achieve better performance in some circumstances. 
To proceed, we first focus on LMI (17) which can be rewritten as

$$
\left[\begin{array}{c}
A \\
I
\end{array}\right]^{T}\left[\begin{array}{cc}
S & 0 \\
0 & -S
\end{array}\right]\left[\begin{array}{c}
A \\
I
\end{array}\right] \prec 0,
$$

and where $S \succ 0$. On the other side, by Schur complement formula followed by congruence transformation and taking $U=S^{-1}$ in the resulting inequality, LMI (17) is equivalent to

$$
\left[\begin{array}{cc}
-U & U A^{T} \\
A U & -U
\end{array}\right] \prec 0
$$

where $U \succ 0$.

\subsubsection{Extension 1}

This paragraph is to be connected to Geromel et al. (1998), Peaucelle et al. (2000).

To introduce extra degree of freedom (by means of additional auxiliary slack variables) we reformulate (22) by application of Lemma 1. To proceed, note that (22) is of the form of the first inequality in (2) where

$$
\Gamma=\left[\begin{array}{cc}
S & 0 \\
0 & -S
\end{array}\right], \quad \Lambda^{\perp}=\left[\begin{array}{c}
A \\
I
\end{array}\right],
$$

then obviously $\Lambda=\left[\begin{array}{ll}-I & A\end{array}\right]$. Furthermore, by taking $\Sigma=I$ the second inequality in (2) vanishes. Now, by invoking Lemma 1 we immediately obtain that LMI (17) is equivalent to

$$
\left[\begin{array}{cc}
S & 0 \\
0 & -S
\end{array}\right]+\operatorname{sym}\left\{\left[\begin{array}{c}
-I \\
A^{T}
\end{array}\right]\left[\begin{array}{ll}
G_{1} & G_{2}
\end{array}\right]\right\} \prec 0,
$$

where $G_{1}$ and $G_{2}$ are additional matrix variables which introduce extra degree of freedom. Because in the above LMI the Lyapunov matrix $S$ is separated from the LRP matrix $A$, this result is particularly useful in the robust context to introduce PDLFs.

\subsubsection{Extension 2}

This paragraph is to be connected to de Oliveira et al. (1999).

Applying the same tools as it has been done for obtaining (25) but with $\Sigma=\left[\begin{array}{l}I \\ 0\end{array}\right]$ give us

$$
\Sigma^{\perp^{T}}=[0 I] .
$$

Hence, taking matrix $\Gamma$ as in (24) again, we get $\Sigma^{\perp^{T}} \Gamma \Sigma^{\perp}=-S \prec 0$. Then, by virtue of Lemma 1, LMI (17) is equivalent to

$$
\left[\begin{array}{cc}
S & 0 \\
0 & -S
\end{array}\right]+\operatorname{sym}\left\{\left[\begin{array}{c}
-I \\
A^{T}
\end{array}\right]\left[\begin{array}{ll}
G_{1} & 0
\end{array}\right]\right\} \prec 0,
$$

where $G_{1}$ is the additional matrix variable. When comparing (26) with (25), we see that enforcing $G_{2}=0$ in (25) does not introduce any additional conservatism (in the nominal case i.e. without uncertainty). 


\subsubsection{Extension 3}

Since for arbitrary chosen real numbers $\rho_{1}$ and $\rho_{2}$ satisfying $\rho_{1}^{2}-\rho_{2}^{2}<0$, the following LMI

$$
\left[\rho_{1} I \quad \rho_{2} I\right]^{T}\left[\begin{array}{cc}
S & 0 \\
0 & -S
\end{array}\right]\left[\begin{array}{l}
\rho_{1} I \\
\rho_{2} I
\end{array}\right]=\left(\rho_{1}^{2}-\rho_{2}^{2}\right) S \prec 0,
$$

holds for $S \succ 0$. Introduce now

$$
\Sigma^{\perp}=\left[\begin{array}{l}
\rho_{1} I \\
\rho_{2} I
\end{array}\right],
$$

and hence we get $\Sigma=\left[-\rho_{2} I \rho_{1} I\right]$. Also, recall the notation of (24) and then an obvious application of Lemma 1 gives that (17) (or equivalently (22)) is feasible when

$$
\left[\begin{array}{cc}
S & 0 \\
0 & -S
\end{array}\right]+\operatorname{sym}\left\{\left[\begin{array}{c}
-I \\
A^{T}
\end{array}\right] G\left[\begin{array}{ll}
-\rho_{2} I & \rho_{1} I
\end{array}\right]\right\} \prec 0,
$$

is solvable for an additional matrix variable $G$.

\subsubsection{Extension 4}

Following the idea presented in Nachidi et al. (2008), let us start with rewriting LMI (23) as

$$
\left[\begin{array}{cc}
-U & U A^{T} \\
A U & -U
\end{array}\right]=\left[\begin{array}{lll}
I & 0 & 0.5 I \\
0 & I & -A
\end{array}\right]\left[\begin{array}{ccc}
0 & 0 & -U \\
0 & -U & 0 \\
-U & 0 & 0
\end{array}\right]\left[\begin{array}{cc}
I & 0 \\
0 & I \\
0.5 I & -A^{T}
\end{array}\right] \prec 0 .
$$

Again, note that the above inequality is of the form of the first inequality in (2) where

$$
\Gamma=\left[\begin{array}{ccc}
0 & 0 & -U \\
0 & -U & 0 \\
-U & 0 & 0
\end{array}\right], \Lambda^{\perp}=\left[\begin{array}{cc}
I & 0 \\
0 & I \\
0.5 I & -A^{T}
\end{array}\right]
$$

then it is obvious that $\Lambda=\left[0.5 I-A^{T}-I\right]$. Next, taking $\Sigma=\left[\begin{array}{lll}-I & 0 & I\end{array}\right]$ we get

$$
\Sigma^{\perp}=\left[\begin{array}{ll}
I & 0 \\
0 & I \\
I & 0
\end{array}\right]
$$

means that the second inequality in (2) is

$$
\Sigma^{\perp^{T}} \Gamma \Sigma^{\perp}=\left[\begin{array}{cc}
-2 U & 0 \\
0 & -U
\end{array}\right] \prec 0,
$$

and obviously always holds for $U \succ 0$. Applying Lemma 1 we immediately obtain that LMI (23) holds if, and only if,

$$
\left[\begin{array}{ccc}
0 & 0 & -U \\
0 & -U & 0 \\
-U & 0 & 0
\end{array}\right]+\operatorname{sym}\left\{\left[\begin{array}{c}
0.5 I \\
-A^{T} \\
-I
\end{array}\right] G\left[\begin{array}{lll}
-I & 0 & I
\end{array}\right]\right\} \prec 0 .
$$

and where the matrix $G$ is an auxiliary slack variable. 


\section{Stabilization of nominal LRP}

Assume that model (9) is subject to the static state feedback control law described by

$$
u_{k+1}(p)=\left[\begin{array}{ll}
K_{1} & K_{2}
\end{array}\right]\left[\begin{array}{c}
x_{k+1}(p) \\
y_{k}(p)
\end{array}\right],
$$

where $K_{1}$ and $K_{2}$ are appropriately dimensioned matrices to be computed. This control law uses feedback of the current state vector (which is assumed to be available for use) and 'feedforward' of the previous pass profile vector. Note that in repetitive processes, the term 'feedforward' is used to describe the case where state or pass profile information from the previous pass (or passes) is used as (part of) the input to a control law applied on the current pass, i.e. to information which is propagated in the pass-to-pass $(k)$ direction. The induced closed-loop model becomes

$$
\begin{aligned}
x_{k+1}(p+1) & =\left(A+B K_{1}\right) x_{k+1}(p)+\left(B_{0}+B K_{2}\right) y_{k}(p), \\
y_{k+1}(p) & =\left(C+D K_{1}\right) x_{k+1}(p)+\left(D_{0}+D K_{2}\right) y_{k}(p) .
\end{aligned}
$$

Assuming that the open-loop process (9) is unstable along the pass, the question is to know if there exists some control law complying with (29) that makes model (30) become stable along the pass. To provide the required result, recall that (26) is the equivalent characterization of (17). Furthermore, LMI (26) can be rewritten as

$$
\left[\begin{array}{cc}
S-G_{1}^{T}-G_{1} & G_{1}^{T} A \\
A^{T} G_{1} & -S
\end{array}\right] \prec 0 .
$$

Since the above LMI is feasible then obviously $S-G_{1}^{T}-G_{1} \prec 0$. This together with $S \succ$ 0 implies that $G_{1}$ is nonsingular and thus invertible. Post- and pre-multiplying the above inequality by

$$
\left[\begin{array}{cc}
G_{1}^{-1} & 0 \\
0 & G_{1}^{-1}
\end{array}\right],
$$

and its transpose, respectively, leads to

$$
\left[\begin{array}{cc}
\widehat{S}-\widehat{G}^{T}-\widehat{G} & A \widehat{G} \\
\widehat{G}^{T} A^{T} & -\widehat{S}
\end{array}\right] \prec 0,
$$

where $\widehat{G}=G_{1}^{-1}$ and $\widehat{S}=\widehat{G}^{T} S \widehat{G}$. Next, for matrices $\Xi_{11}, \Xi_{12}, \Xi_{22}$ which form a matrix $\Xi$ as in (5), define

$$
\widehat{\Xi}_{11}=\widehat{G}^{T} \Xi_{11} \widehat{G}, \widehat{\Xi}_{12}=\widehat{G}^{T} \Xi_{12} \widehat{G}, \widehat{\Xi}_{22}=\widehat{G}^{T} \Xi_{22} \widehat{G}
$$

which implies that matrices $P$ and $Q$ in (6)-(8) have to be replaced with $\widehat{P}$ and $\widehat{Q}$ respectively, where

$$
\widehat{P}=\widehat{G}^{T} P \widehat{G}, \widehat{Q}=\widehat{G}^{T} Q \widehat{G} .
$$

Now, along the notation (32) and (33) we have the following sufficient condition for the existence of controller (29) for discrete LRPs.

Theorem 2 Suppose that a discrete LRP as decribed by (9) is subject to a control law of the form (29). Then the resulting closed-loop LRP (30) is stable along the pass over a finite 
frequency range $\theta \in \Theta$ if there exist matrices $\widehat{S} \succ 0, \widehat{P} \succ 0, \widehat{Q} \succ 0, N_{1}, N_{2}$ and $\widehat{G}$ such that the following LMIs

$$
\begin{gathered}
{\left[\begin{array}{cc}
\widehat{S}-\widehat{G}^{T}-\widehat{G} & A \widehat{G}+B N_{1} \\
\widehat{G}^{T} A^{T}+N_{1}^{T} B^{T} & -\widehat{S}
\end{array}\right] \prec 0,} \\
{\left[\begin{array}{cccc}
\widehat{\Xi}_{11} & \widehat{\Xi}_{12}-\widehat{G}^{T} & 0 & 0 \\
\widehat{\Xi}_{12}^{*}-\widehat{G} & \widehat{\Xi}_{22}+\operatorname{sym}\left\{A \widehat{G}^{T}+B N_{1}\right\} & B_{0}+B N_{2} & \widehat{G}^{T} C^{T}+N_{1}^{T} D^{T} \\
0 & B_{0}^{T}+N_{2}^{T} B^{T} & -I & D_{0}^{T}+N_{2}^{T} D^{T} \\
0 & C \widehat{G}+D N_{1} & D_{0}+D N_{2} & -I
\end{array}\right] \prec 0,}
\end{gathered}
$$

hold and where matrices $\widehat{\Xi}_{11}, \widehat{\Xi}_{12}, \widehat{\Xi}_{22}$ are defined in (32) and chosen according to the specific frequency range $\Theta$ by the same manner as in Theorem 1. Also, if LMIs (31) and (35) are feasible, the required control law matrices $K_{1}$ and $K_{2}$ of (29) can be computed using

$$
K_{1}=N_{1} \widehat{G}^{-1}, K_{2}=N_{2} .
$$

Proof In view of Theorem 1 it can be shown that the closed-loop LRP (30) is stable along the pass if the following inequalities are satisfied

$$
\left(A+B K_{1}\right)^{T} S\left(A+B K_{1}\right)-S \prec 0,
$$

$$
\left[\begin{array}{cccc}
\Xi_{11} & \Xi_{12}-W & 0 & 0 \\
\Xi_{12}^{*}-W^{T} & \Xi_{22}+\operatorname{sym}\left\{W^{T}\left(A+B K_{1}\right)\right\} & W^{T}\left(B_{0}+B K_{2}\right) & \left(C+D K_{1}\right)^{T} \\
0 & \left(B_{0}+B K_{2}\right)^{T} W & -I & \left(D_{0}+D K_{2}\right)^{T} \\
0 & C+D K_{1} & D_{0}+D K_{2} & -I
\end{array}\right] \prec 0
$$

for $S \succ 0, P \succ 0, Q \succ 0$ and $W$. Then, according to the developed results, take (31) as the equivalence to (37) where $A$ is replaced with $A+B K_{1}$. Next, setting $N_{1}=K_{1} \widehat{G}$ in the resulting inequality we directly obtain (34). Furthermore, rewrite (38) with $W=\widehat{G}^{-1}$ and apply the congruence transformation with $\operatorname{diag}\{\widehat{G}, \widehat{G}, I, I\}$. It yields the LMI (35) where $N_{2}=K_{2}$.

Remark 2 Some level of conservatism can be introduced by taking $W=\widehat{G}^{-1}$, i.e. by choosing the same auxiliary slack variable in both LMIs (34) and (35).

Remark 3 The LMI (34) can be replaced by another LMI obtained from different LMI extensions presented in the previous section.

It is interesting to note that the matrices $K_{1}$ and $K_{2}$ are separated from Lyapunov matrices which bring additional flexibility, reducing conservatism, especially in the uncertain case.

\section{Robust stability and stabilization of LRPs}

As it has been indicated in Introduction, the interest in introducing auxiliary slack variables is not only to bring flexibility in the design but also to enable the implicit involvement of PDLFs when the model is itself parameter-dependent. 
Indeed, assume that the state-space model matrices of (9) involve unknown but fixed uncertainties described by polytopies. This means that all process matrices are actually dependent on a real parameter vector $\xi$ with a polytopic dependency:

$$
\left[\begin{array}{lll}
A & B_{0} & B \\
C & D_{0} & D
\end{array}\right] \in \Omega:=\left\{\left[\begin{array}{lll}
A(\xi) & B_{0}(\xi) & B(\xi) \\
C(\xi) & D_{0}(\xi) & D(\xi)
\end{array}\right]=\sum_{i=1}^{N} \xi_{i}\left[\begin{array}{lll}
A_{i} & B_{0 i} & B_{i} \\
C_{i} & D_{0 i} & D_{i}
\end{array}\right], \quad \xi \in \mathscr{D}\right\}
$$

where

$$
\mathscr{D}=\left\{\xi=\left[\begin{array}{c}
\xi_{1} \\
\vdots \\
\xi_{N}
\end{array}\right]: \xi_{i} \geq 0, \sum_{i=1}^{N} \xi_{i}=1\right\} .
$$

It means that the various matrices $A_{i}, B_{0 i}, B_{i}, C_{i}, D_{0 i}, D_{i}$ define the vertices of a polytope in which the actual matrices $A, B_{0}, B, C, D_{0}$ and $D$ lie.

Definition 1 The discrete LRP described by (9) where the process matrices are subject to uncertainty as in (39) and (40) is robustly stable along the pass if, and only if, it is stable along a pass for any value of $\xi$ in $\mathscr{D}$.

Now assume that matrix functions $P(\xi), Q(\xi)$ and $S(\xi)$, which are to be determined, are expected to have the following forms

$$
P(\xi)=\sum_{i=1}^{N} \xi_{i} P_{i}, Q(\xi)=\sum_{i=1}^{N} \xi_{i} Q_{i}, S(\xi)=\sum_{i=1}^{N} \xi_{i} S_{i},
$$

and are positive definite for all values of $\xi$ such that

$$
\left[\begin{array}{lll}
A & B_{0} & B \\
C & D_{0} & D
\end{array}\right] \in \Omega
$$

The matrices defined in (41) are PDLFs and can take $N$ different values corresponding to the vertices of the polytope $\Omega$. According to PDLFs $P(\xi)$ and $Q(\xi)$ of the form (41), matrices $\Xi_{11}, \Xi_{12}, \Xi_{22}$ which form a matrix $\Xi$ as in (5) have affine form

$$
\Xi_{11}(\xi)=\sum_{i=1}^{N} \xi_{i} \Xi_{11 i}, \quad \Xi_{12}(\xi)=\sum_{i=1}^{N} \xi_{i} \Xi_{12 i}, \quad \Xi_{22}(\xi)=\sum_{i=1}^{N} \xi_{i} \Xi_{22 i}
$$

Along the above notation, we have the following result.

Theorem 3 Suppose that a discrete LRP of the form (9) is subject to uncertainty structure modeled by (39) and (40). Then this LRP is stable along the pass over a finite frequency range $\theta \in \Theta$ for all admissible uncertainties if there exist matrices $S_{i} \succ 0, Q_{i} \succ 0, P_{i} \succ 0$, $W$ and $G_{1}$ such that the following LMIs are feasible

$$
\begin{aligned}
& {\left[\begin{array}{cc}
S_{i}-G_{1}^{T}-G_{1} & G_{1}^{T} A_{i} \\
A_{i}^{T} G_{1} & -S_{i}
\end{array}\right] \prec 0,} \\
& {\left[\begin{array}{cccc}
\Xi_{11 i} & \Xi_{12 i}-W & 0 & 0 \\
\Xi_{12}{ }_{i}^{*}-W^{T} & \Xi_{22 i}+A_{i}^{T} W+W^{T} A_{i} & W^{T} B_{0 i} & C_{i}^{T} \\
0 & B_{0 i}^{T} W & -I & D_{0 i}^{T} \\
0 & C_{i} & D_{0 i} & -I
\end{array}\right] \prec 0,}
\end{aligned}
$$


for all $i=1, \ldots, N$, and where according to Lemma 2 and notation (42) the matrices $\Xi_{11 i}$, $\Xi_{12 i}, \Xi_{22 i}$ are chosen according to the specific frequency range $\Theta$, i.e. for low, middle or high frequency range, respectively.

The most interesting aspect of the above stability condition is that it can be easily exploited to cope with the problem of state feedback robust stabilization where we are interested to find matrices $K_{1}$ and $K_{2}$ of (29) such that the closed-loop process is robustly stable along the pass over a finite frequency range $\theta \in \Theta$. To proceed, observe that by introducing PDLFs in (41) the matrices defined in (32) and (33) also become parameter-dependent, i.e.

$$
\widehat{P}(\xi)=\sum_{i=1}^{N} \xi_{i} \widehat{P}_{i}, \widehat{Q}(\xi)=\sum_{i=1}^{N} \xi_{i} \widehat{Q}_{i}, \widehat{S}(\xi)=\sum_{i=1}^{N} \xi_{i} \widehat{S}_{i},
$$

and hence

$$
\widehat{\Xi}_{11}(\xi)=\sum_{i=1}^{N} \xi_{i} \widehat{\Xi}_{11 i}, \widehat{\Xi}_{12}(\xi)=\sum_{i=1}^{N} \xi_{i} \widehat{\Xi}_{12 i}, \widehat{\Xi}_{22}(\xi)=\sum_{i=1}^{N} \xi_{i} \widehat{\Xi}_{22 i} .
$$

Now, we have the following result which gives the sufficient condition for a discrete LRP to be robustly stable along the pass under control law of (29).

Theorem 4 Suppose that the control law of the form (29) is applied to a discrete LRP (9) with associated uncertainty structure (39) and (40). Then the resulting closed-loop LRP (30) is robustly stable along the pass over a finite frequency range $\theta \in \Theta$ if there exist matrices $\widehat{S}_{i} \succ 0, \widehat{P}_{i} \succ 0, \widehat{Q}_{i} \succ 0, N_{1}, N_{2}$ and $\widehat{G}$ such that the following LMIs

$$
\begin{aligned}
& {\left[\begin{array}{cc}
\widehat{S}_{i}-\widehat{G}^{T}-\widehat{G} & A_{i} \widehat{G}+B N_{1} \\
\widehat{G}^{T} A_{i}^{T}+N_{1}^{T} B^{T} & -\widehat{S}_{i}
\end{array}\right] \prec 0,}
\end{aligned}
$$

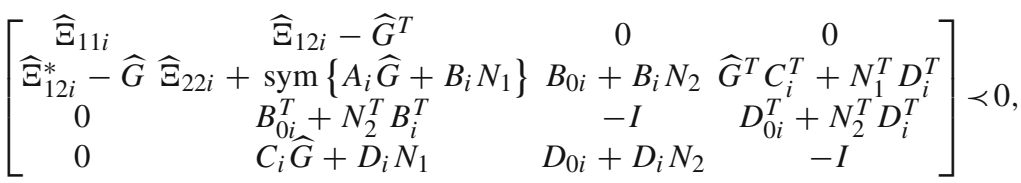

hold for all $i=1, \ldots, N$ and where the matrices $\widehat{\Xi}_{11 i}, \widehat{\Xi}_{12 i}, \widehat{\Xi}_{22 i}$ are defined by (46) and chosen according to the specific frequency range $\Theta$ by the same manner as in Theorem 1. Also, if LMIs (31) and (35) are feasible, the required control law matrices $K_{1}$ and $K_{2}$ of (29) can be calculated using (36).

Proof The proof can be completed in an identical manner to the proof of Theorem 2 where we use PDLFs defined in (45) and (46).

\section{Numerical example}

To illustrate our result, consider the robust stabilization problem for a metal rolling process using the approach given in Theorem 4.

It is known that metal rolling is an extremely common industrial process where, in essence, deformation of the workpiece takes place between two rolls with parallel axes revolving in opposite directions. The metal strip is to be rolled to a pre-specified thickness by passing it through a series of rolls for successive reductions. In practice, a number of models of this 
process can be developed depending on the assumptions made on the underlying dynamics and the particular mode of operation under consideration. Here, however, we will restrict attention to a linearized model of the dynamics adopted from Cichy et al. (2011) presented as the discrete LRP with uncertainty structure of (39) and (40) where

$$
\begin{aligned}
& {\left[\begin{array}{c|c|c}
A_{1} & B_{01} & B_{1} \\
\hline C_{1} & D_{01} & D_{1}
\end{array}\right]=\left[\begin{array}{cc|c|c}
0.9377 & 187.5361 & 0.0144 & -0.0866 \\
-3.116 \cdot 10^{-4} & 0.9377 & 7.1907 \cdot 10^{-5} & -4.3278 \cdot 10^{-4} \\
\hline 0.9377 & 187.5361 & 0.7836 & -0.0866
\end{array}\right],} \\
& \begin{array}{l}
{\left[\begin{array}{l|l|l}
A_{2} & B_{02} & B_{2} \\
\hline C_{2} & D_{02} & D_{2}
\end{array}\right]=\left[\begin{array}{cc|c|c}
0.7676 & 153.5191 & 0.0536 & -0.0709 \\
-1.162 \cdot 10^{-3} & 0.7676 & 2.6816 \cdot 10^{-4} & -3.5427 \cdot 10^{-4} \\
\hline 0.7676 & 153.5191 & 0.8229 & -0.0709
\end{array}\right],} \\
{\left[\begin{array}{l|l|l}
A_{3} & B_{03} & B_{3} \\
\hline C_{3} & D_{03} & D_{3}
\end{array}\right]=\left[\begin{array}{cc|c|c}
0.8574 & 171.481 & 0.0823 & -0.198 \\
-7.1297 \cdot 10^{-4} & 0.8574 & 4.1172 \cdot 10^{-4} & -9.9024 \cdot 10^{-4} \\
\hline 0.8574 & 171.481 & 0.5049 & -0.198
\end{array}\right],}
\end{array} \\
& {\left[\begin{array}{l|l|l}
A_{4} & B_{04} & B_{4} \\
\hline C_{4} & D_{04} & D_{4}
\end{array}\right]=\left[\begin{array}{cc|c|c}
0.925 & 185.0033 & 4.6328 \cdot 10^{-3} & -0.0229 \\
-3.7492 \cdot 10^{-4} & 0.925 & 2.32 \cdot 10^{-5} & -1.143 \cdot 10^{-4} \\
\hline 0.925 & 185.0033 & 0.9428 & -0.0229
\end{array}\right] .}
\end{aligned}
$$

The above model has been obtained from differential LRP model given in Bochniak et al. (2008) after discretization procedure with sampling time $T=0.2 \mathrm{~s}$. Then performing the design procedure of Theorem 4 in MATLAB for low frequency range where $\theta_{l}=0.3770$ (recall that $\theta_{l}=2 \pi \omega T$ and we take $\omega=0.3 \mathrm{~Hz}$ ) gives the corresponding controller matrices of (29) as

$$
K_{1}=\left[\begin{array}{lll}
2.4693 & 1408.0368
\end{array}\right], K_{2}=1.7531 .
$$

It is easy to show that the eigenvalues of $\left(A_{i}+B_{i} K_{1}\right)$ and $D_{0 i}+D_{i} K_{2}$ for $i=1,2,3,4$ are located within the unit circle. Also, it can be checked that for the considered frequency range from 0 to $0.3 \mathrm{~Hz}$ the controlled processes at each vertex of polytope (called Process 1 , Process 2, Process 3 and Process 4 for $N=1,2,3,4$ respectively) are stable along the pass. To confirm it, it is sufficient to plot singular value plot-see Fig. 1, to see the frequency response of controlled processes are less than $1(0 \mathrm{~dB})$ for frequencies from 0 to $0.3 \mathrm{~Hz}$.

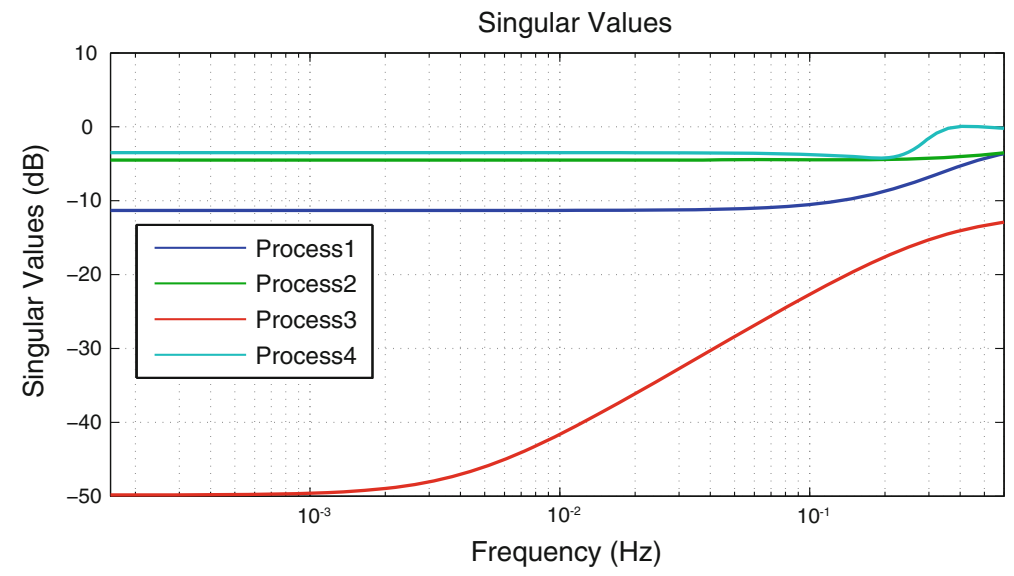

Fig. 1 Singular value plots for the controlled processes 


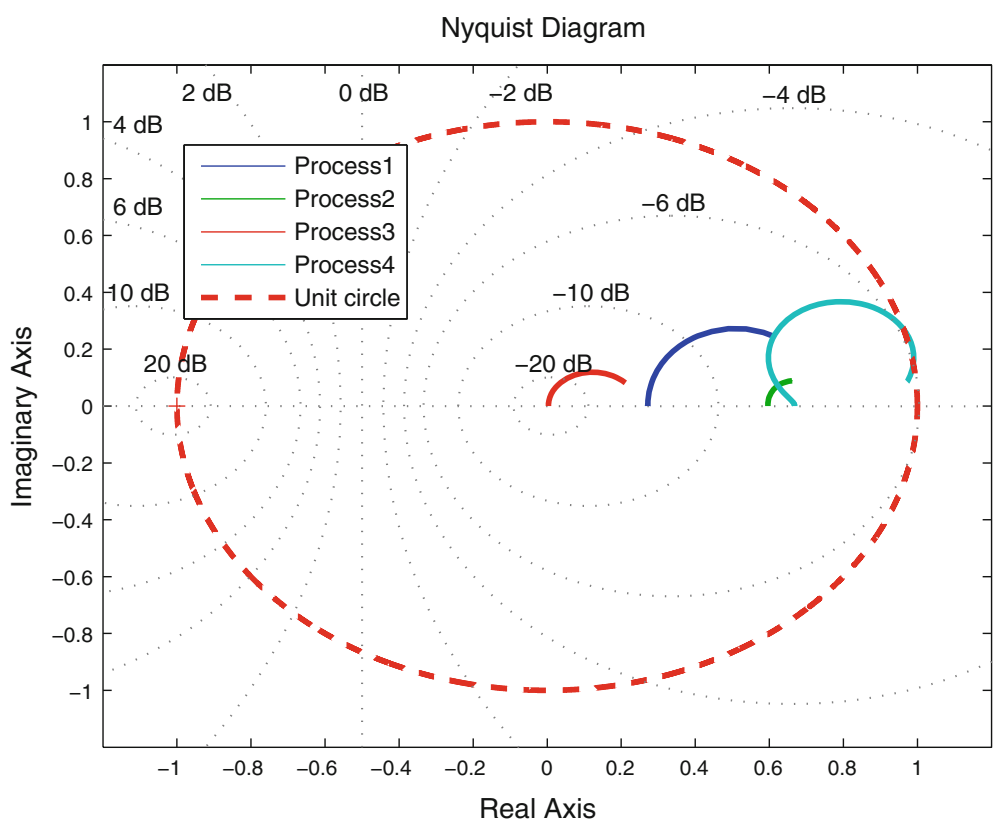

Fig. 2 Nyquist plots for the controlled processes

Also, due to the fact that the considered processes are SISO then stability along the pass of controlled processes over considered frequency range can be confirmed with Nyquist plots see Fig. 2.

When comparing the obtained result with those presented in Cichy et al. (2011), we see that controller gain matrices computed over entire frequency range have more large gains than these obtained here over finite frequency range only and therefore presented approach gives some practical implementation advantages. Also, it should be emphasized that due to polynomial time complexity of the considered LMI problems, the efficient solution to the considered controller design problem can be even maintained for higher problem dimensions. In particular, the overall number of decision variables in (47) and (48) is

$$
3 N \frac{n(n+1)}{2}+n^{2}+m n+m r,
$$

where according to (9) $n$ denotes the number of states, $m$ is the number of outputs, $r$ is the number of inputs and $N$ is the vertices number of the polytope. This means that the considered examples involve computations with 43 decision variables and this is more than corresponding result of Cichy et al. (2011). The difference is mostly caused by using 2 more parameter dependent matrix variables in LMIs (47) and (48).

\section{Conclusions}

This paper has developed substantial new results on the relatively open problem of robust control of LRPs which are a distinct class of 2D linear systems of both systems theoretic and applications interest. Particularly, it has been shown that it is possible to introduce an extra 
matrix variable which allows us to separate the Lyapunov matrices from the process matrices and then give us a way to derive LMI conditions based on parameter dependent Lyapunov functions. Also it has been shown that this analysis and controller design procedures can be extended to the case of a finite frequency domain. These results have great practical potential and can be used for many engineering applications. The theoretical findings have been illustrated by the numerical example. It would be interesting to extend the results of this paper to the cases of the dynamic pass profile synthesis. Another obvious extension would be to consider the LRPs model with time-varying polytopic uncertainties such as in Daafouz and Bernussou (2001), more sophisticated uncertainty structures (such as norm-bounded Linear Implicit Fractional Representations (ILFR) as in Sari et al. (2011)) or also to use more complex PDLFs (Chesi et al. 2005, 2007; Olivieira et al. 2008; Olivieira and Peres 2006). This could be investigated in a near future.

Open Access This article is distributed under the terms of the Creative Commons Attribution License which permits any use, distribution, and reproduction in any medium, provided the original author(s) and the source are credited.

\section{References}

Azevedo-Perdicoulis, T.-P., \& Jank, G. (2012). Disturbance attenuation of linear quadratic OL-Nash games on repetitive processes with smoothing on the gas dynamics. Multidimensional Systems and Signal Processing, 23(1), 131-153.

Bachelier, O., Henrion, D., Pradin, B., \& Mehdi, D. (2004). Robust matrix root-clustering of a matrix in intersections or unions of subregions. SIAM Journal of Control and Optimization, 43(3), 1078-1093.

Bachelier, O., \& Mehdi, D. (2006). Robust matrix root-clustering through extended KYP Lemma. SIAM Journal of Control and Optimization, 45(1), 368-381.

Bachelier, O., Paszke, W., \& Mehdi, D. (2008). On the KYP lemma and the multidimensional models. Multidimensional Systems and Signal Processing, 19(3-4), 425-447.

Bochniak, J., Gałkowski, K., \& Rogers, E. (2008). Multi-machine operations modelled and controlled as switched linear repetitive processes. International Journal of Cotrol, 81(10), 1549-1567.

Boland, F. M., \& Owens, D. H. (1980). Linear multipass processes: A two-dimensional interpretation. In IEE Proceedings, Part D-control theory and applications, vol. 127, no. 5, pp. 189-193.

Boyd, S., El Ghaoui, L., Feron, E., \& V. Balakrishnan (1994). Linear matrix inequalities in system and control theory, ser. SIAM studies in applied and numerical mathematics, vol. 15. Philadelphia, USA: SIAM.

Bristow, D. A., Tharayil, M., \& Alleyne, A. (2006). A survey of iterative learning control. IEEE Control Systems Magazine, 26(3), 96-114.

Chesi, G., Garulli, A., Tesi, A., \& Vicino, A. (2005). Polynomially parameter-dependent Lyapunov functions for robust stability of polytopic systems: an LMI approach. IEEE Transactions on Automatic Control, 50(3), 365-370.

Chesi, G., Garulli, A., Tesi, A., \& Vicino, A. (2007). Robust stability of time-varying polytopic systems via parameter-dependent homogeneous Lyapunov functions. Automatica, 43(2), 309-316.

Cichy, B., Augusta, P., Rogers, E., Gałkowski, K., \& Hurak, Z. (2008). On the control of distributed parameter systems using a multidimensional systems setting. Mechanical Systems and Signal Processing, 22, 15661581.

Cichy, B., Gałkowski, K., Rogers, E., \& Kummert, A. (2011). An approach to iterative learning control for spatio-temporal dynamics using nD discrete linear systems models. Multidimensional Systems and Signal Processing, 22, 83-96.

Cichy, B., Gałkowski, K., Rogers, E., \& Kummert, A. (2011). Numerical Linear Algebra in signals, systems and control, ser. Lecture notes in electrical engineering, ch. Robust Control of Discrete Linear Repetitive Processes with Parameter Varying Uncertainty, vol. 80, Springer.

Daafouz, J., \& Bernussou, J. (2001). Parameter dependent Lyapunov functions for discrete time systems with time varying parametric uncertainties. Systems and Control Letters, 43(5), 355-359.

de Oliveira, M. C., Bernussou, J., \& Geromel, J. C. (1999). A new discrete-time robust stability condition. Systems and Control Letters, 37(4), 261-265. 
El Ghaoui, L., \& Niculescu, S.-I. (1999). Advances in linear matrix inequality methods in control, ser. Advances in Design and Control, Philadelphia, vol. 2. USA: SIAM.

Feng, Y., Yagoubi, M., \& Chevrel, P. (2010). Dilated LMI characterisations for linear time-invariant singular systems. International Journal of Control, 83(11), 2276-2284.

Gahinet, P., \& Apkarian, P. (1994). A linear matrix inequality approach to $\mathscr{H}_{\infty}$ control. International Journal of Robust and Nonlinear Control, 4, 421-448.

Gahinet, P., Apkarian, P., \& Chilali, M. (1996). Affine parameter-dependent Lyapunov functions and real parametric uncertainty. IEEE Transactions on Automatic Control, 41(3), 436-442.

Geromel, J. C., de Oliveira, M. C., \& Hsu, L. (1998). LMI characterization of structural and robust stability. Linear Algebra and its Applications, 285(1-3), 69-80.

Hmamed, A., Alfidi, M., Benzaouia, A., \& Tadeo, F. (2008). LMI conditions for robust stability of 2D linear discrete-time systems. Mathematical Problems in Engineering, vol. 2008, 2008.

Huang, T. S. (1972). Stability of two-dimensional and recursive filters. IEEE Transactions on Audio and Electroacustics, $A U-20,158-163$.

Iwasaki, T., \& Hara, S. (2005). Generalized KYP lemma: unified frequency domain inequalities with design applications. IEEE Transactions on Automatic Control, 50(1), 41-59.

Iwasaki, T., \& Hara, S. (2007). Feedback control synthesis of multiple frequency domain specifications via generalized KYP lemma. International Journal of Robust and Nonlinear Control, 17, 415-434.

Nachidi, M., Tadeo, F., Hmamed, A., \& Alfidi, M. (2008). Static output-feedback controller design for twodimensional Roesser models. International Journal of Sciences and Techniques of Automatic control \& computer engineering, 2(2), 738-749.

Olivieira, R. C. L. F., de Oliveira, M. C., \& Peres, P. L. D. (2008) Convergent LMI relaxations for robust analysis of uncertain linear systems using lifted polynomial parameter-dependent lyapunov functions. Systems and Control Letters, 57(8), 680-689.

Olivieira, R. C. L. F., Peres, P. L. D. (2006). LMI conditions for robust stability analysis based on polynomially parameter-dependent Lyapunov functions. Systems and Control Letters, 55(1), 52-61.

Paszke, W., \& Bachelier, O. (2009) New robust stability and stabilization conditions for linear repetitive processes. In Proceedings of the international workshop on multidimensional (nD) systems, nDS 2009, pp. 1-6, Thessaloniki, Greece, June 29-July 12009.

Paszke, W., Gałkowski, K., Rogers, E. (2012). Repetitive process based iterative learning control design using frequency domain analysis. In Proceedings of the the 2012 IEEE multi-conference on systems and control (MSC 2012), Dubrovnik, Croatia, 3-6 October 2012.

Paszke, W., Rapisarda, P., Rogers, E., \& Steinbuch, M. (2009). Dissipative stability theory for linear repetitive processes with application in iterative learning control. In Proceedings symposium on learning control at IEEE CDC 2009, Shanghai, China, 16-18 December 2009.

Paszke, W., Rogers, E., \& Gałkowski, K. (2011). Design of robust iterative learning control schemes in a finite frequency range. In Proceedings of the international workshop on multidimensional (nD) systems, $n D S$ 2011, pp. 1-6, Poitiers, France, 5-7 September 2011.

Peaucelle, D., \& Arzelier, D. (2001). Robust performance analysis with LMI-based methods for real parametric uncertainty via parameter-dependent Lyapunov functions. IEEE Transactions on Automatic Control, 46(4), 624-630.

Peaucelle, D., Arzelier, D., Bachelier, O., \& Bernussou, J. (2000). A new robust D-stability condition for real convex polytopic uncertainty. Systems and Control Letters, 40(1), 21-30.

Pipeleers, G., Demeulenaere, B., Swevers, J., \& Vandenberghe, L. (2009). Extended LMI characterizations for stability and performance of linear systems. Systems and Control Letters, 58(7), 510-518.

Rabenstein, R., \& Steffen, P. (2012). Numerical iterative methods and repetitive processes. Multidimensional Systems and Signal Processing, 23(1-2), 163-183.

Ramos, D. C. W., \& Peres, P. L. D. (2001). A less conservative LMI condition for robust stability of discretetime uncertain systems. Systems and Control Letters, 43(5), 371-378.

Rogers, E., Gałkowski, K., \& Owens, D. H. (2007). Control systems theory and applications for linear repetitive processes, ser. Lecture notes in control and information sciences, vol. 349. Berlin, Germany: Springer .

Sari, B., Bachelier, O., \& Mehdi, D. (2011). Robust $S$-regularity of matrix pencils applied to the analysis of descriptor models. Linear Algebra and its Applications, 435(5), 923-942.

Trofino, A. (1999). Parameter-dependent Lyapunov functions for a class of uncertain linear systems: An LMI approach. In Proceedings of the 38th IEEE conference decision and control (CDC), vol. 1, pp. 2341-2346. Phoenix, USA, 7-10 December 1999.

Wu, L., Xiaojie, S., \& Peng, S. (2011). Mixed $\mathscr{H}_{2} / \mathscr{H}_{\infty}$ approach to fault detection of discrete linear repetitive processes. Journal of the Franklin Institute, 348(2), 393-414. 


\section{Author Biographies}

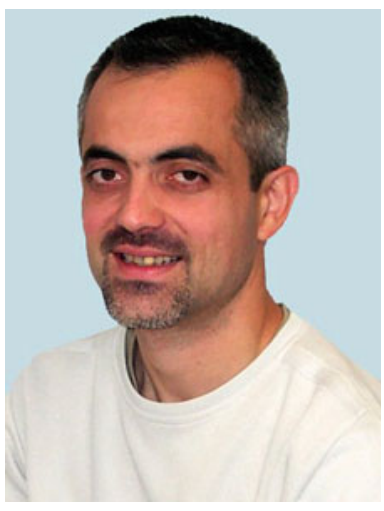

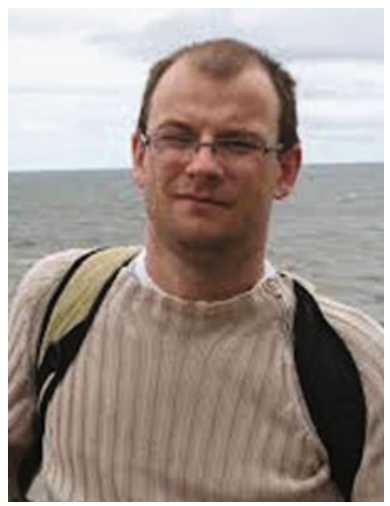

Wojciech Paszke was born in Zielona Gora, Poland, in 1975. He received his M.Sc. and Ph.D. degrees, both in electrical engineering, from the Technical University of Zielona Gora, in 2000 and 2005, respectively. Between 2008 and 2010 he was affiliated to Eindhoven University of Technology, The Netherlands, where he has been a control systems expert on high precision positioning of electron microscope. Currently, he is affiliated to the Institute of Control and System Engineering at the University of Zielona Gora, Poland. His research is focused on multidimensional $(\mathrm{nD})$ systems, repetitive processes, iterative learning control schemes and convex optimization in robust control problems.

Olivier Bachelier was born in Châteauroux, France, in 1971. He received the Engineer's Degree and the Masters' Degree with specialization in control from the INSA (Institut National des Sciences Appliquées), Toulouse, France, in September 1994. From December 1995 to September 1998, he was a Ph.D. student at the LAAS (Laboratoire d'Analyse et d'Architecture des Systémes) of the CNRS (Centre National de la Recherche Scientifique) in Toulouse, France. He received the Ph.D. degree from INSA in September 1998. He kept on working for one year at the LAAS-CNRS. From December 1995 to September 1999, he also worked as a teacher in Control and Computer Science at INSA. In September 1999, he joined the IUT (Institut Universitaire de Technologie) of Poitiers-Châtellerault-Niort and the LIAS (Laboratoire d'Informatique et d'Automatique pour les Systèmes) which depends on the ENSIP (Ecole Nationale Supérieure d'Ingénieurs de Poitiers) and on the ENSMA (Ecole Nationale Supérieure de Mécanique et d'Aérotechnique) in Poitiers, where he has been working up to now. He received the habilitation in March 2009 and was appointed professor in September 2012, still in the IUT (the IUT and the ENSIP are parts of the University of Poitiers). Olivier Bachelier is studying robust analysis and robust design of uncertain multi-input multiouput linear models. He is particularly interested in robust matrix root-clustering, pole and eigenstructure assignment, descriptor systems and multidimensional models. 